\title{
A One-step-ahead Information-based Feedback Control for Binaural Active Localization
}

\author{
Gabriel Bustamante, Patrick Danès, Thomas Forgue and Ariel Podlubne \\ LAAS-CNRS, Université de Toulouse, CNRS, UPS, Toulouse, France - Email: firstname.name@laas.fr
}

\begin{abstract}
Fundamental limitations of binaural localization, such as front-back ambiguity or distance non-observability, can be overcome by combining the sensed audio signals with the sensor motor commands into "active" schemes. Such strategies can rely on stochastic filtering. In this context, this paper addresses the determination of an admissible motion of a binaural head leading, on average, to the one-step-ahead most informative localization. To this aim, a constrained optimization problem is set up, which consists in maximizing the entropy of the next predicted measurement probability density function over a cylindric admissible set. The proposed optimum policy is validated on real-life robotic experiments.
\end{abstract}

\section{INTRODUCTION}

The advent of auditory robots has led to the emergence of audio-motor binaural localization schemes that, by combining binaural perception and motion, can disambiguate front from back and recover source range [1][2]. Given such an audiomotor localization strategy (e.g., [3]), the question remains how to drive the robot so as to gather as much spatial information on a source as possible.

In Robotics, Simultaneous Localization and Mapping (SLAM) techniques have been extended to cope with the neighboring "exploration problem", i.e., to design robot motions improving their knowledge about the environment [4]. Control policies could be found by maximizing information criteria related to the robot situation, e.g., by determining the direction of maximum local information improvement. Shannon entropy or mutual information have often been used [5], as well as the Fisher information matrix (FIM) [6]. It has been shown that a mapping robot guided by a mutual information based controller can be attracted towards unexplored areas [7]. Similar strategies have been used to coordinate multiple sensor platforms [8]. Information-theoretic controllers can address different objectives such as the control of a robot-mounted camera to optimize depth estimation [9], or the sensor parameters selection (e.g. zoom or attitude) for scene analysis [10], [11]. In the bearings-only tracking problem, optimum receiver maneuvers could be determined by maximizing a cost functional involving FIM determinants [12].

In robot audition, auditory scene exploration has also been investigated [13]. A mobile robot equipped with a microphone array localizes sound sources and estimates its own position in a known geometric map [14]. Motion planning based on audio situation has been proposed to improve speech recognition by a monaural robot [15]. In other studies, sound source localization was improved by optimizing the position of microphones deployed in the environment [16]. In [17], a robot equipped with a microphone array was controlled to locate a sound source by minimizing a criterion based on the entropy of an occupancy grid used to represent the source position belief.

Considering a prior knowledge on the relative position of a static sound source with respect to a binaural head (e.g., spherical or anthropomorphic), the determination of an admissible finite motion of the sensor is studied, which leads to the minimum uncertainty in the one-step-ahead localization. This paper therefore complements [18], which was inherently limited to infinitesimal displacements, and could not address optimality among all admissible motions.

\section{PRoblem STATEMENT AND ORGANIZATION OF THE PAPER}

A frame $\mathcal{F}=\left(O, \overrightarrow{x_{R}}, \overrightarrow{y_{R}}, \overrightarrow{z_{R}}\right)$ is attached to a binaural sensor, where: $O$ stands for the midpoint of the two microphones $R_{1}, R_{2} ; \overrightarrow{z_{R}}$ points to boresight; $\overrightarrow{y_{R}}$ supports the interaural axis; $\overrightarrow{x_{R}}$ is vertical and points downwards. Between two consecutive times $k$ and $k+1$, the frame $\mathcal{F}_{k}=\left(O_{k}, \overrightarrow{x_{R}}, \overrightarrow{y_{R}}, \overrightarrow{z_{R}}\right)$ is turned into $\mathcal{F}_{k+1}=\left(O_{k+1},{\overrightarrow{x_{R}}}_{k+1},{\overrightarrow{y_{R}}}_{k+1}, \overrightarrow{z_{R}} \overrightarrow{x+1}\right)$ by means of the translation $T=T_{y} \overrightarrow{y_{R}}{ }_{k}+T_{z} \overrightarrow{z_{k}}$ followed by the rotation of angle $\phi \triangleq\left(\overrightarrow{z_{R}}, \overrightarrow{z_{R}}{ }_{k+1}\right)$ around $\overrightarrow{x_{R k}}$. The pointwise source $E$ and $R_{1}, R_{2}$ are supposed to stay on the same horizontal plane. So, the vector $x=\left(e_{x}, e_{y}, e_{z}\right)^{T}$ depicting the Cartesian coordinates of the source in the (moving) sensor frame obeys the state equation

$$
x_{k+1}=R^{T}(\phi) x_{k}-R^{T}(\phi) T+w_{k},
$$

with $w_{k}$ the dynamic noise and $R(\phi)$ the rotation matrix corresponding to $\phi$.

A belief on the sensor-to-source position $x_{k}=\left(e_{y}, e_{z}\right)^{T}$ at time $k$ is given in terms of the 2D Gaussian pdf $\mathcal{N}\left(x_{k} ; \hat{x}_{k \mid k}, P_{k \mid k}\right)$, with $\hat{x}_{k \mid k}$ its estimate and $P_{k \mid k}$ the associated error covariance. $\hat{x}_{k \mid k}$ and $P_{k \mid k}$ can be the posterior mean and covariance obtained by fusing into a stochastic filtering scheme the short-term spatial information extracted from the binaural stream with the motion of the sensor, along [3]. The problem dealt with in this paper consists in determining the motion of the sensor which best improves, on average, the next localization of the sound source. First, a metrics is described, uniting the belief on the state at time $k$ and the rigid motion applied over $[k ; k+1]$, to the expected information obtained after a measurement update at time $k+1$. To make the problem 
tractable, this measurement update guiding the exploration relies on a closed form equation such as

$$
z_{k}=l\left(x_{k}\right)+v_{k}=\bar{l}\left(\theta_{k}\right)+v_{k}, z_{k} \in \mathbb{R}, v_{k} \sim \mathcal{N}\left(0, R_{k}\right),
$$

with $v_{k}$ the measurement noise and $\theta_{k}=-\operatorname{atan} 2\left(e_{y}, e_{z}\right)$ the relative azimuth of the source ( 0 along $\overrightarrow{z_{R}}$ and increasing clockwise). Considering a farfield source, $\bar{l}($.$) can express the$ genuine time delay of arrival between a pair of microphones in free field, or the Woodworth-Schlosberg ITD approximation between antipodal microphones over a spherical head [19], etc.

A constrained optimization problem is first defined, whose solution leads to the next best sensor position. Then, useful insights into the geometry of the problem are provided. Experiments conclude the paper.

\section{INFORMATION FEEDBACK CONTROL}

\section{A. An information-theoretic constrained optimization problem}

Let $x, y$ be continuous random variables with joint and marginal pdfs $p(x, y)$ and $p(x), p(y)$. The differential entropy $h(x)$ embodies the uncertainty in $x$, and the mutual information $I(x, y) \geq 0$ measures the amount of information that $p(x)$ contains about $p(y)$. They are defined by [20]

$$
\begin{aligned}
h(x) & =-\int p(x) \log p(x) \mathrm{d} x \\
I(x, y) & =\iint p(x, y) \log \frac{p(x, y)}{p(x) p(y)} \mathrm{d} x \mathrm{~d} y .
\end{aligned}
$$

When conditioned on the event that a random variable $z$ takes a given value, they are henceforth denoted by $h(x \mid z)$, $h(y \mid z)$ and $I(x, y \mid z)$. The conventional Bayes rule underlying the measurement update stage relates the next filtered state pdf $p\left(x_{k+1} \mid z_{1: k+1}\right)$, the next predicted state pdf $p\left(x_{k+1} \mid z_{1: k}\right)$, the observation model $p\left(z_{k+1} \mid x_{k+1}\right)$ and the next predicted measurement pdf $p\left(z_{k+1} \mid z_{1: k}\right)$. Consequently, entropies and mutual information of these distributions can be connected with an entropy update rule [21]. The expectation

$$
\iint-\log p\left(x_{k+1} \mid z_{1: k+1}\right) p\left(x_{k+1} ; z_{k+1} \mid z_{1: k}\right) \mathrm{d} x_{k+1} \mathrm{~d} z_{k+1}
$$

of $-\log p\left(x_{k+1} \mid z_{1: k+1}\right)$ conditioned on $z_{1: k}$, which is also equal to $\mathrm{E}_{z_{k+1}}\left\{h\left(x_{k+1} \mid z_{1: k+1}\right)\right\}$, satisfies [18]

$$
\begin{aligned}
\mathrm{E}_{z_{k+1}}\left\{h\left(x_{k+1} \mid z_{1: k+1}\right)\right\} & =h\left(x_{k+1} \mid z_{1: k}\right)-I\left(x_{k+1} ; z_{k+1} \mid z_{1: k}\right) \\
\mathrm{E}_{x_{k+1}}\left\{h\left(z_{k+1} \mid x_{k+1}\right)\right\} & =h\left(z_{k+1} \mid z_{1: k}\right)-I\left(x_{k+1} ; z_{k+1} \mid z_{1: k}\right),
\end{aligned}
$$

with $I\left(x_{k+1} ; z_{k+1} \mid z_{1: k}\right)$ the conditional mutual information of the next state and measurement. As $I\left(x_{k+1} ; z_{k+1} \mid z_{1: k}\right) \geq 0$, $\mathrm{E}_{z_{k+1}}\left\{h\left(x_{k+1} \mid z_{1: k+1}\right)\right\} \leq h\left(x_{k+1} \mid z_{1: k}\right)$ holds, which stresses the information gain brought by the measurement update.

Let $|$.$| term the determinant of a matrix. If w_{k}$ is negligible in (1), then $h\left(x_{k+1} \mid z_{1: k}\right)=\frac{1}{2} \log \left[(2 \pi e)^{n}\left|P_{k+1 \mid k}\right|\right]$ is also equal to $\frac{1}{2} \log \left[(2 \pi e)^{n}\left|P_{k \mid k}\right|\right]$ in view of the fact that the sensor undergoes a rigid motion (the vector $R^{T}(\phi) T$ is constant in (1), so $P_{k+1 \mid k}=R^{T}(\phi) P_{k \mid k} R(\phi)$ with $\left.|R(\phi)|=1\right)$. In addition, $h\left(z_{k+1} \mid x_{k+1}\right)=\frac{1}{2} \log \left[(2 \pi e)^{n}\left|R_{k+1}\right|\right]$ is also independent of the control variables $T, \phi$. Besides, the (nonlinear)
Kalman measurement update equations at time $k+1$ show that both $h\left(x_{k+1} \mid z_{1: k+1}\right)=\frac{1}{2} \log \left[(2 \pi e)^{n}\left|P_{k+1 \mid k+1}\right|\right]$ and $h\left(z_{k+1} \mid z_{1: k}\right)=\frac{1}{2} \log \left[(2 \pi e)^{n}\left|S_{k+1 \mid k}\right|\right]$ do not depend on the measurement $z_{k+1}$. Consequently, the following rule holds.

Theorem 1: Finding the next best sensor position, induced by the rigid body motion $\left(T^{*}, \phi^{*}\right)$, which minimizes the entropy $h\left(x_{k+1} \mid z_{1: k+1}\right)$ of the next filtered state $p d f$ is equivalent to maximizing the mutual information $I\left(x_{k+1} ; z_{k+1} \mid z_{1: k}\right)$ of the next predicted state and measurement, or to maximizing the entropy $h\left(z_{k+1} \mid z_{1: k}\right)$ of the next predicted measurement pdf. In other words, $\left(T^{*}, \phi^{*}\right)$ is the solution of

$$
(\mathcal{P})\left\{\begin{aligned}
\left(T^{*}, \phi^{*}\right)= & \underset{(T, \phi) \in \mathcal{T} \times \mathcal{R}}{\arg \min } h\left(x_{k+1} \mid z_{1: k+1}\right) \\
= & \underset{(T, \phi) \in \mathcal{T} \times \mathcal{R}}{\arg \max } I\left(x_{k+1} ; z_{k+1} \mid z_{1: k}\right) \\
= & \underset{(T, \phi) \in \mathcal{T} \times \mathcal{R}}{\arg \max } h\left(z_{k+1} \mid z_{1: k}\right) .
\end{aligned}\right.
$$

where $\mathcal{T}$ and $\mathcal{R}$ respectively term the sets of admissible translations and rotations.

\section{B. Interpretation}

From (2), the loci of the sensor-to-source positions $x$ corresponding to iso-values of the measurement $z$ are radial lines rigidly linked to the frame $\mathcal{F}$ and passing through $O$. For TDOA/ITD measurements, these loci are not uniformly distributed along the azimuths. They are more concentrated along the direction of $\overrightarrow{z_{R}}$ which defines the auditive fovea, while they are sparser along the interaural axis $\overrightarrow{y_{R}}$.

Given a belief $\mathcal{N}\left(x_{k} ; \hat{x}_{k \mid k}, P_{k \mid k}\right)$ on the head-tosource position at time $k$, Figure 1 sketches the 2D Gaussian approximation of the next filtered state pdf $\mathcal{N}\left(x_{k+1} ; \hat{x}_{k+1 \mid k+1}, P_{k+1 \mid k+1}\right)$ after applying various rigid motions $(T, \phi)$ to the sensor. All the involved normal distributions are depicted by related 99\%-probability confidence ellipses. Importantly, if the dynamic noise is neglected in (1), then the next predicted state pdf $\mathcal{N}\left(x_{k+1} ; \hat{x}_{k+1 \mid k}, P_{k+1 \mid k}\right)$ is basically described by the same ellipse as for the initial belief, but "viewed" from the sensor once it has completed its motion. Equation (2) implies that the spatial information brought by the measurement $z_{k+1}$ can be described by a $99 \%$-probability confidence cone tapering to the apex $O_{k+1}$. For a given variance $R$ of the measurement noise, the extent of this cone on either side of the line corresponding to the genuine azimuth of the source is all the more important as the iso- $z$ loci are sparse.

From the initial configuration on Figure 1a, the head first undergoes a pure rotational motion so that the auditive fovea (supported by $\overrightarrow{z_{R+1}}$ ) becomes oriented towards the wide axis of the confidence ellipse associated to the next predicted state pdf (Figure 1b). On Figure 1c, translation and rotation are applied so as to drive $O_{k+1}$ on the line supported by the small axis of that ellipse, and a subsequent rotation makes $\vec{y}_{k+1}$ point towards its center. Last, in Figure 1d, the auditive fovea $\vec{z}_{R+1}$ is driven towards the small axis of that ellipse.

The variance $S_{k+1 \mid k}$ of the predicted measurement pdf is heuristically related to the number of iso- $z$ loci intersecting the 


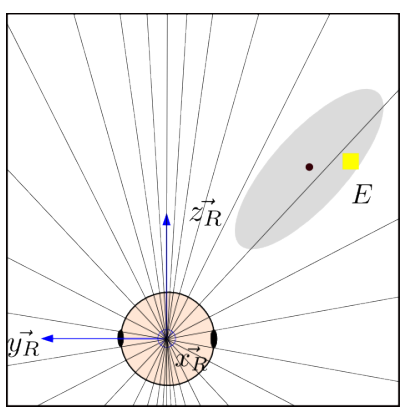

(a)

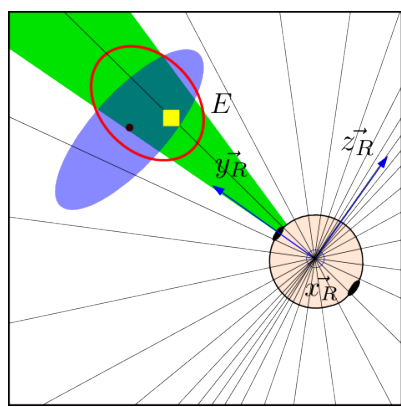

(c)

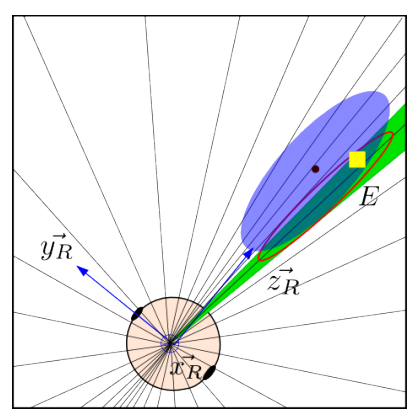

(b)

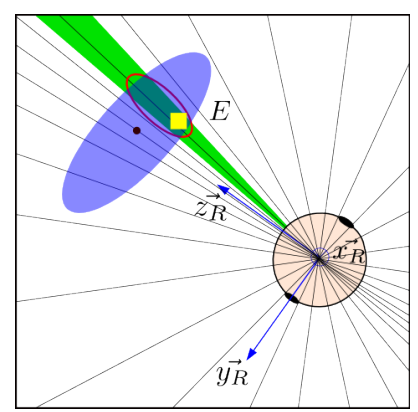

(d)
Fig. 1: (a): Frame $\mathcal{F}_{k}$ attached to the binaural head (blue); sound source genuine position (yellow square); confidence ellipse associated to the belief at time $k$ (grey); iso- $z_{k}$ loci depicting the measurement space (grey radial lines). (b)-(c)(d): Frame $\mathcal{F}_{k+1}$ (blue); confidence ellipse associated to the next predicted state pdf at time $k+1$ (blue); iso- $z_{k+1}$ loci (grey); confidence cone associated to the measurement (green); confidence ellipse associated to the next filtered state pdf (i.e., the next belief at $k+1$ ), after the incorporation of $z_{k+1}$ (red).

confidence ellipse associated to the predicted state pdf. The more iso- $z$ loci intersect that ellipse, the higher is $S_{k+1 \mid k}$.

As aforementioned, the confidence cone describing the spatial uncertainty due to the measurement noise is wide if the source lies along the interaural axis (Figure 1c). In this case, a small number of iso- $z$ loci intersect the confidence ellipse associated to the predicted state pdf, so that the measurement update cannot significantly improve the information in the filtered state pdf. When the auditive fovea is oriented towards the confidence ellipse, the measurement uncertainty is narrower, the variance $S_{k+1 \mid k}$ is important and the measurement update is more efficient (Figures 1b-1d). It is even better when the fovea points to the small axis of the ellipse (Figure 1d). Importantly, the spatial uncertainty due to the measurement noise shrinks as the head gets closer to the source, what leads to an increase in the predicted measurement variance.

\section{Numerical solution via the projected gradient algorithm}

In view of the above, starting from the head-to-source position belief $\mathcal{N}\left(x_{k} ; \hat{x}_{k \mid k}, P_{k \mid k}\right)$ at time $k$, the adequate optimum finite translations $\left(T_{y}^{*}, T_{z}^{*}\right)$ and rotations $\phi^{*}$ maximize the log-determinant (here, simply the value) of the (co)variance $S_{k+1 \mid k}=F_{k}\left(T_{y}, T_{z}, \phi\right)$ of the next predicted measurement pdf, with $F_{k}: \mathbb{R}^{3} \rightarrow \mathbb{R}$. Then, the optimization problem $(\mathcal{P})$ can be formulated as

$$
(\mathcal{P})\left\{\begin{aligned}
&\left(T_{y}^{*}, T_{z}^{*}, \phi^{*}\right)=\underset{T_{y}, T_{z}, \phi}{\arg \max } F_{k}\left(T_{y}, T_{z}, \phi\right) \\
& \text { subject to } f_{1}: \phi-\phi_{\max } \leq 0 \\
& \\
& f_{2}:-\phi-\phi_{\max } \leq 0 \\
& f_{3}: T_{y}{ }^{2}+T_{z}{ }^{2}-r_{\max } \leq 0
\end{aligned}\right.
$$

with $r_{\max }$ and $\phi_{\max }$ the maximum admissible translation and rotation. The admissible values of the decisions variables thus constitute a cylinder volume. Due to the geometry of the problem depicted in the previous section, the optimum solution(s) can be shown to lie on the external surface of this admissible set. So, the last inequality constraint is turned into its limiting counterpart $f_{3}=0$.

Let the position on the cylinder surface be defined by two parameters $(\alpha, \phi)$ with

$$
\begin{aligned}
g: \mathbb{R}^{2} & \rightarrow \mathbb{R}^{3} \\
\left(\begin{array}{l}
\alpha \\
\phi
\end{array}\right) & \mapsto\left(\begin{array}{l}
g_{1}(\alpha, \phi) \\
g_{2}(\alpha, \phi) \\
g_{3}(\alpha, \phi)
\end{array}\right)=\left(\begin{array}{c}
r \sin (\alpha) \\
r \cos (\alpha) \\
\phi
\end{array}\right) .
\end{aligned}
$$

Then the solution of $(\mathcal{P})$ with $f_{3}=0$ is equivalent to solve

$\left(\mathcal{P}^{\prime}\right):\left(\alpha^{\star}, \phi^{\star}\right)=\max _{\alpha, \phi} \tilde{F}_{k}(\alpha, \phi)$ s.t. $\left\{\begin{array}{l}\tilde{f}_{1}: \phi-\phi_{\max } \leq 0 \\ \tilde{f}_{2}:-\phi-\phi_{\max } \leq 0\end{array}\right.$

with $\tilde{F}_{k}(.,)=.\left[F_{k} \circ g\right](.,$.$) and \tilde{f}_{1}, \tilde{f}_{2}$ the expression of $f_{1}, f_{2}$ in terms of the new set of decision variables.

The projected gradient algorithm is used to solve the problem numerically. Though $F_{k}$ has no closed form, an analytic approximation of its gradient around $D=\left(T_{y}, T_{z}, \phi\right)^{T}$ can be derived by using chained first order Taylor expansions and the unscented transform. This leads to

$$
F_{k}(D+d u)=F_{k}(D)+\nabla F_{k}(D)^{T} d u,
$$

with $d u=\left(d T_{y}, d T_{z}, d \phi\right)^{T}$ the infinitesimal motion vector applied around $D$ and $\mathrm{d}_{k}=\nabla F_{k}(D)$ the gradient of $F_{k}$ at $D$. Then the gradient $\tilde{\mathrm{d}}_{k}=\nabla \tilde{F}_{k}(\alpha, \phi)$ can be expressed as

$$
\nabla \tilde{F}_{k}(\alpha, \phi)=\nabla F_{k}(g(\alpha, \phi)) \frac{\partial g(\alpha, \phi)}{\partial\left(\begin{array}{c}
\alpha \\
\phi
\end{array}\right)^{T}}
$$

and used in the projected gradient algorithm.

\section{Geometric CUes}

In this section, $\bar{l}($.$) in (2) consists in the Woodworth-$ Schlosberg formula for ITD approximation over a spherical head [19], i.e., $\bar{l}(\theta)=\frac{a}{c}(\theta+\sin (\theta))$ for $|\theta| \in\left[0, \frac{\pi}{2}\right], \quad \bar{l}(\theta)=\frac{a}{c}(\pi-\theta+\sin (\theta)) \quad$ for $\theta \in\left[\frac{\pi}{2}, \pi\right]$ and $\bar{l}(\theta)=\frac{a}{c}(-\pi-\theta+\sin (\theta))$ for $\theta \in\left[-\pi,-\frac{\pi}{2}\right]$. Its iso- $z_{k}$ loci are similar to those depicted in Figure 1. The belief $\mathcal{N}\left(x_{k} ; \hat{x}_{k \mid k}, P_{k \mid k}\right)$ on the sensor-to-source position at time $k$ is such that $\hat{x}_{k \mid k}=(1,1.5)^{T}$. The uncertainty on the source location, depicted by $P_{k \mid k}$, is associated to a confidence region which is either ellipsoidal or circular. The function $S_{k+1 \mid k}=F_{k}(., ., \phi)$ has been evaluated w.r.t. $T=T_{y} \overrightarrow{y R}_{k}+T_{z} \overrightarrow{z_{k} k}$ for three different subsequent rotations 
$\phi$ of the head. The iso-values of $F_{k}(., ., \phi)$ have been displayed in the plane defined by the current local frame $\mathcal{F}_{k}$. The set of admissible translations has been represented as well as the constrained local maximum. When there is no rotation (Figure 2a), the contour lines become distorted for $T=(1, .)^{T}$ and $T=(., 1.5)^{T}$. These distortions are the consequences of the geometric considerations evoked in $\S$ III-B. For $T=(1, .)^{T}$ (resp. $\left.T=(., 1.5)^{T}\right), O_{k+1}$ would be aligned with the major axis of the ellipse (resp. the interaural axis would become aligned to the small axis of the ellipse). The given rotations of the head $\left(+45^{\circ}\right.$ (Figure $\left.2 \mathrm{~b}\right)$ and $-45^{\circ}$ (Figure 2c)) lead to the rotation of the contour lines and change the local maximum. Hence, it is more attractive in this situation to apply a rotation of $-45^{\circ}$ than $+45^{\circ}$. It is worth noting that the distortion for $T=(1, .)^{T}$ vanishes when the confidence region is circular (Figure $2 \mathrm{~d}$ ).

Then the function $\tilde{F}_{k}(.,$.$) has been evaluated for the$ same prior belief. It appears that the maximum is located on $\phi=-48^{\circ}$. It corresponds to the maximum value of the function $F_{k}(., .,$.$) , on the edge of the cylinder.$

\section{REAL EXPERIMENTS}

Live experiments have been conducted in a moderately reverberant open-space environment. A mobile robot supports a KEMAR binaural head and torso form G.R.A.S. ${ }^{\circledR}$, whose neck is endowed with a controllable dof. The position of the source and of the robot are measured in real time by an Optitrack ${ }^{\mathrm{TM}}$ motion capture system, with $\pm 0.1 \mathrm{~mm}$ accuracy. The robot software architecture is based on the ROS middleware and the GenoM3 components generator [22]. The task which manages the program, plots and saves the results, is performed by a MATLAB ${ }^{\circledR}$ client.

The localization process is divided into three steps. First, a likelihood function of the source azimuth is determined from the binaural stream. A white noise filtered with a $1 \mathrm{kHz}-$ bandwidth $1 \mathrm{kHz}$-central-frequency bandpass filter is selected as the source signal, so as to get a smooth likelihood. Then, this short-term directional cue is combined with the motor commands of the robot to compute a Gaussian mixture approximation of the posterior pdf [23]. This posterior pdf is reduced to a single Gaussian $\mathcal{N}\left(x_{k} ; \hat{x}_{k \mid k}, P_{k \mid k}\right)$ by keeping its most probable hypothesis, and the likelihood function is traded for the Woodworth-Schlosberg measurement equation to compute the exploratory control inputs of the robot. The whole process is repeated every $T_{s}=1 \mathrm{~s}$.

The results of the localization as well as the genuine position of the source are displayed in real-time (Figures $3 a-3 c \& 3 e-3 g$ ). A comparison with open-loop motion strategies, namely a rotation of the head and a translation along its interaural axis, is reported on Figure 3d. Videos are available at http://homepages.laas.fr/danes/EUSIPCO2016.

\section{CONCLUSION}

This paper has presented a method to actively improve the binaural localization of a sound source. It has been assessed on an embedded auditory platform and compared to other motion

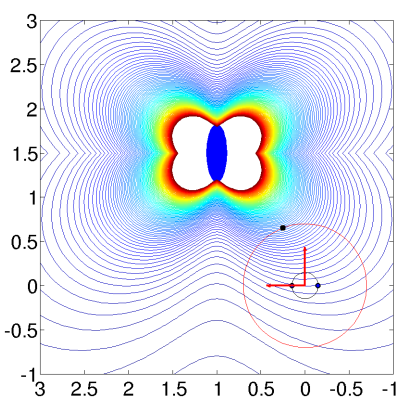

(a)

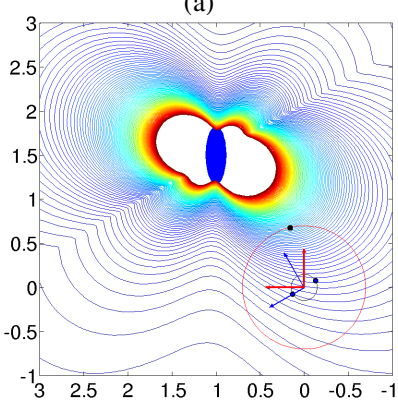

(c)

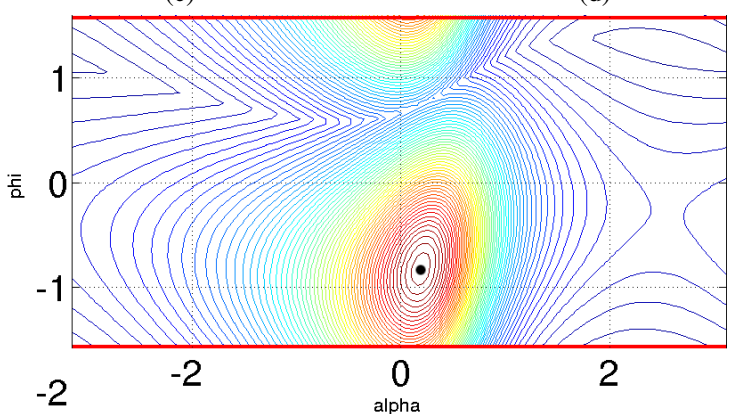

(e)

Fig. 2: Iso-values of the function $F_{k}(., ., \phi)$ (a-b-c-d) and $\tilde{F}_{k}(.,$.$) (e) - The sensor frame \mathcal{F}_{k}=\left(O, \overrightarrow{x_{R}}, \overrightarrow{y_{R}}, \overrightarrow{z_{R}}\right)$ is plotted in red and the blue ellipses/circle represent the $99 \%$ confidence region of the sound source location extracted from the belief $\mathcal{N}\left(x_{k} ; \hat{x}_{k \mid k}, P_{k \mid k}\right)$. Axes are in meters. The red circle delimits the admissible translation $T \in \mathcal{T}$. The contours are warm (resp. cold) when $S_{k+1}$ is important (resp. low). The red axes on (e) represent the $\pm 90^{\circ}$-limits of the head rotation.

strategies. Future work will consist in investigating $N$-stepahead information-based control. One difficulty of the problem may lie in defining an additive criterion which expresses the average information measure to be optimized at the end of a $N$-element sliding temporal window.

\section{ACKNOWLEDGMENT}

This work was partially supported by EU FET Grant Two!Ears, ICT-618075, www.twoears.eu.

\section{REFERENCES}

[1] M. Cooke, Y. Lu, Y. Lu, and R. Horaud, "Active hearing, active speaking," in Int. Symp. on Auditory and Audiological Research (ISAAR'07), Marienlyst, Helsigør, Denmark, 2007.

[2] K. Nakadai, T. Lourens, H. Okuno, and H. Kitano, "Active audition for humanoid," in Nat. Conf. on Artificial Intelligence (AAAI'2000), Austin, TX, 2000. 


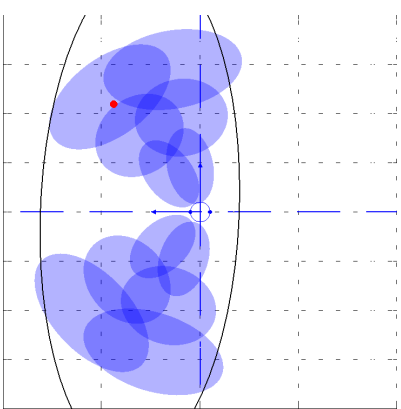

(a)

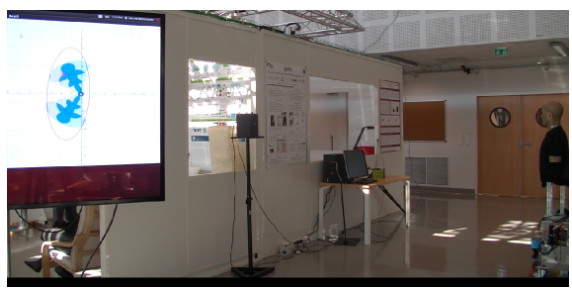

(e)

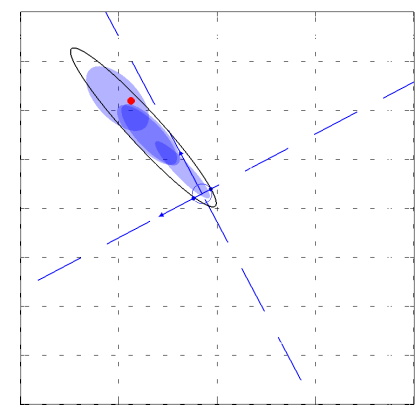

(b)

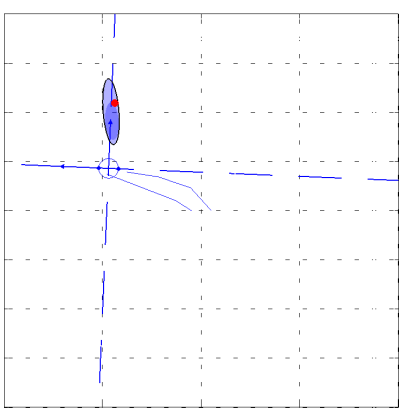

(c)

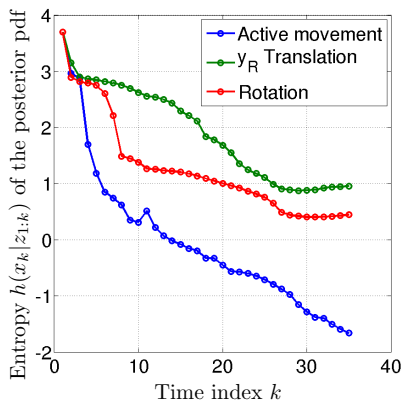

(d)

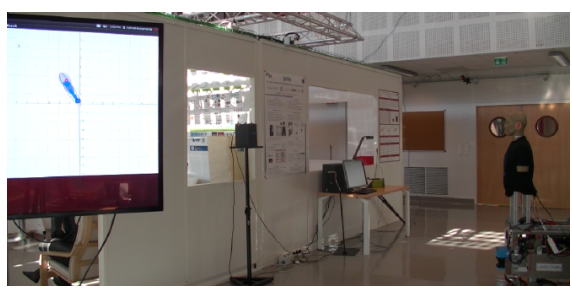

(f)

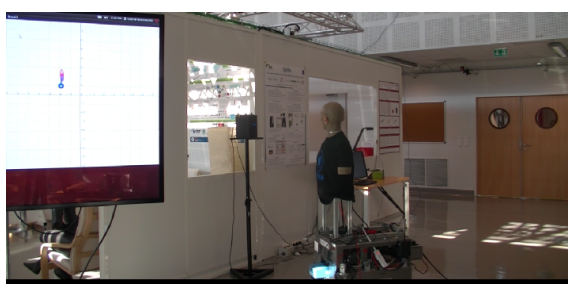

(g)

Fig. 3: Active motion for a single source localization - 99\%-probability confidence ellipses associated to each hypothesis of the sensor-to-source (Gaussian mixture) posterior pdf are represented by the blue ellipses w.r.t. a reference frame linked to the world. Screenshots of the recorded video are reported at times: $2 \mathrm{~s}(\mathrm{a} \& \mathrm{e}), 10 \mathrm{~s}(\mathrm{~b} \& \mathrm{f})$ and $34 \mathrm{~s}(\mathrm{c} \& \mathrm{~g})$. On these plots, the grid spacings are $2 \mathrm{~m}$ and $1 \mathrm{~m}$ along the horizontal and vertical axis, respectively. Figure (d) compares the obtained entropy $h\left(x_{k} \mid z_{1: k}\right)$ for various motion strategies.

[3] A. Portello, P. Danès, and S. Argentieri, "Active binaural localization of intermittent moving sources in the presence of false measurements," in IEEE/RSJ Int. Conf. on Intell. Robots and Systems (IROS'2012), 2012.

[4] S. Thrun, W. Burgard, and D. Fox, Probabilistic Robotics. The MIT Press, 2005.

[5] F. Bourgault, A. Makarenko, S. Williams, B. Grocholsky, and H. Durrant-Whyte, "Information based adaptive robotic exploration," in IEEE/RSJ Int. Conf. on Intelligent Robots and Systems, (IROS'2002), Lausanne, Switzerland, 2002.

[6] H. Feder, J. Leonard, and C. Smith, "Adaptive mobile robot navigation and mapping," The International Journal of Robotics Research, vol. 18, no. 7, pp. 650-668, 1999.

[7] B. Julian, "Mutual information-based gradient-ascent control for distributed robotics," Ph.D. dissertation, Massachusetts Institute of Technology, 2013.

[8] B. Grocholsky, A. Makarenko, and H. Durrant-Whyte, "Informationtheoretic coordinated control of multiple sensor platforms," in IEEE Int. Conf. on Robotics and Automation, (ICRA'03), Taipei, Taiwan, 2003.

[9] C. Forster, M. Pizzoli, and D. Scaramuzza, "Appearance-based active, monocular, dense reconstruction for micro aerial vehicles," in Proceedings of Robotics: Science and Systems, Berkeley, USA, July 2014.

[10] J. Denzler and C. Brown, "Information theoretic sensor data selection for active object recognition and state estimation," IEEE Trans. on Pattern Analysis and Machine Intelligence, vol. 24, no. 2, pp. 145-157, 2002.

[11] E. Sommerlade and I. Reid, "Information-theoretic active scene exploration," in IEEE Conf. on Computer Vision and Pattern Recognition, (CVPR'2008), Anchorage, Alaska, 2008.

[12] J.-P. Le Cadre and S. Laurent-Michel, "Optimizing the receiver maneuvers for bearings-only tracking," Automatica, vol. 35, no. 4, pp. 591-606, 1999.

[13] E. Martinson and A. Schultz, "Discovery of sound sources by an autonomous mobile robot," Autonomous Robots, vol. 27, pp. 221-237, Oct. 2009.

[14] Y. Sasaki, S. Thompson, M. Kaneyoshi, and S. Kagami, "Mapgeneration and identification of multiple sound sources from robot in motion," in IEEE/RSJ Intl. Conf. Intell. Robots and Systems (IROS'2010), Taipei, Taiwan, 2010, pp. 437-443.
[15] M. Kumon, K. Fukushima, S. Kunimatsu, and M. Ishitobi, "Motion planning based on simultaneous perturbation stochastic approximation for mobile auditory robots," in IEEE/RSJ Int. Conf. on Intelligent Robots and Systems (IROS'2010), Taipei, Taiwan, 2010.

[16] E. Martinson, T. Apker, and M. Bugajska, "Optimizing a reconfigurable robotic microphone array," in IEEE/RSJ Int. Conf. on Intelligent Robots and Systems (IROS'2011), San Francisco, California, 2011.

[17] E. Vincent, A. Sini, and F. Charpillet, "Audio source localization by optimal control of a mobile robot," in IEEE Int. Conf. on Acoustics, Speech and Signal Processing (ICASSP'2015), Brisbane, Australia, 2015.

[18] G. Bustamante, P. Danès, T. Forgue, and A. Podlubne, "Toward information-based feedback control for binaural active localization," in IEEE Int. Conf. on Acoustics, Speech, and Signal Processing (in press ICASSP'2016), Shanghai, China, 2016.

[19] N. Aaronson and W. Hartmann, "Testing, correcting, and extending the woodworth model for interaural time difference," The Journal of the Acoustical Society of America, vol. 135, pp. 817-823, 2014.

[20] T. Cover and J. Thomas, Elements of Information Theory. Wiley, 1991.

[21] J. Manyika, "An information-theoretic approach to data fusion and sensor management," Ph.D. dissertation, University of Oxford, 1993.

[22] A. Mallet, C. Pasteur, M. Herrb, S. Lemaignan, and F. Ingrand, "Genom3: Building middleware-independent robotic components," in IEEE Int. Conf. on Robotics and Automation, (ICRA'2010), Anchorage, Alaska, 2010.

[23] A. Portello, G. Bustamante, P. Danès, J. Piat, and J. Manhès, "Active localization of an intermittent sound source from a moving binaural sensor," in Forum Acustium (FA'2014), Krakow, Poland, 2014. 